03;04

\title{
Параметры микроканальной структуры в начальной фазе искрового разряда в воздухе атмосферного давления в промежутке острие-плоскость
}

\author{
(C) К.И. Алмазова ${ }^{1}$, А.А. Амирова ${ }^{2}$, А.Н. Белоногов ${ }^{1}$, В.В. Боровков ${ }^{1}$, Г.Б. Рагимханов ${ }^{3,}$, \\ Д.В. Терешонок ${ }^{4}$, А.А. Тренькин ${ }^{1}$, 3.P. Халикова ${ }^{3}$ \\ ${ }^{1}$ Российский федеральный ядерный центр - Всероссийский научно-исследовательский институт экспериментальной \\ физики, Саров, Россия \\ ${ }^{2}$ Институт физики Дагестанского федерального исследовательского центра РАН, Махачкала, Россия \\ ${ }^{3}$ Дагестанский государственный университет, Махачкала, Россия \\ ${ }^{4}$ Объединенный институт высоких температур РАН, Москва, Россия \\ ฯ E-mail: gb-r@mail.ru
}

Поступило в Редакцию 31 августа 2020г.

В окончательной редакции 5 октября 2020 г.

Принято к публикации 5 октября 2020 г.

\begin{abstract}
Представлены результаты исследований микроструктуры в начальной фазе искрового разряда в воздухе в промежутке острие-плоскость длиной $1.5 \mathrm{~mm}$. Измерения показывают, что в течение $15 \mathrm{~ns}$ после пробоя канал представляет собой совокупность большого числа микроканалов, ток в канале нарастает практически линейно до значения $1 \mathrm{kA}$, а концентрация электронов достигает значения $2 \cdot 10^{19} \mathrm{~cm}^{-3}$. С учетом экспериментальных данных проведены расчеты кинетики процессов в отдельном микроканале. Установлено, что средняя температура электронов составляет от 4 до $8 \mathrm{eV}$, напряженность электрического поля $\sim 300 \mathrm{kV} / \mathrm{cm}$, электропроводность $\sim 10 \Omega^{-1} \cdot \mathrm{cm}^{-1}$. Полученные результаты свидетельствуют о том, что именно микроструктура разряда обусловливает относительно высокие значения средней температуры электронов в совокупности с достаточно большой степенью ионизации.
\end{abstract}

Ключевые слова: газовый разряд, микроструктура, температура электронов, степень ионизации газа.

DOI: 10.21883/PJTF.2021.02.50541.18533

Актуальность активного исследования импульсных газовых разрядов обусловлена их широким практическим применением. Несмотря на длительный период изучения, для полноценного понимания и описания некоторых аспектов газоразрядных процессов все еще недостаточно экспериментальных данных. Особенно это касается начальной фазы разрядов в плотных газах ввиду необходимости высокого пространственно-временно́го разрешения регистрирующего оборудования и методик [1].

Относительно недавно с помощью теневых и интерференционных методов было обнаружено, что в начальной фазе искрового разряда визуально выглядящий сплошным канал может представлять собой пучок, состоящий из большого числа микроканалов [2,3].

В настоящей работе представлены экспериментальные данные о начальной фазе микроструктурированного искрового разряда в промежутке острие-плоскость в воздухе атмосферного давления, а также результаты расчетно-теоретического рассмотрения процессов в отдельных микроканалах в интервале времени от 2 до $15 \mathrm{~ns}$ после пробоя. Работа дополняет результаты исследований [3], которые посвящены рассмотрению динамики искры при временах 20-60 ns.

С практической точки зрения это может представлять существенный интерес для создания плазмохимических реакторов.
Эксперименты проводились на стенде, включающем генератор импульсов напряжения (ГИН), кабельную линию, разрядный промежуток, диагностическую аппаратуру и систему синхронизации. Подробно аппаратура и методики описаны в работе [3] (см. также ссылки к ней).

Импульс напряжения с ГИН (амплитуда $30 \mathrm{kV}$, длительность фронта $7 \mathrm{~ns}$, полярность положительная) через кабельную линию длиной $7 \mathrm{~m}$ подавался на разрядный промежуток острие-плоскость с зазором $1.5 \mathrm{~mm}$. Параметры острийного электрода были следующими: диаметр $10 \mathrm{~mm}$, угол при вершине $14^{\circ}$, радиус кривизны $0.1 \mathrm{~mm}$, материал - нержавеющая сталь. Плоский электрод был изготовлен из алюминиевого сплава.

Измерение напряжения (емкостным делителем) и тока (резистивным шунтом) осуществлялось на выходе ГИН с временны́ разрешением не хуже $1 \mathrm{~ns}$.

Система оптической регистрации включала в себя источник зондирующего излучения - лазер (длина волны $\lambda=532 \mathrm{~nm}$, длительность импульса на полувысоте $6 \mathrm{~ns}$ ), объектив, светофильтры и цифровую электронно-оптическую камеру, посредством которой после прохождения лазерного излучения через область разряда регистрировалось его изображение. Пространственное разрешение системы регистрации не хуже $5 \mu \mathrm{m}$. 




Рис. 1. Фрагмент осциллограмм напряжения $U$ и тока $I$ на выходе ГИН, соответствующий начальной стадии разряда. $t_{U}$ - момент появления напряжения на разрядном промежутке, $t_{b d}-$ момент времени, принятый за начало пробоя.

С применением данной системы регистрации и оптической схемы на базе интерферометра Маха-Цендера была реализована интерференционная методика. Экспозиция кадра интерферограммы определялась длительностью импульса лазера. Интерференционная методика позволяла определять усредненную по сечению концентрацию электронов в канале разряда с погрешностью не более $20 \%$.

Сдвигом момента запуска системы оптической регистрации относительно момента пробоя обеспечивалась визуализация различных стадий разрядного процесса. Режим съемки однокадровый (один кадр за импульс).

На рис. 1 приведен фрагмент осциллограмм напряжения $U$ и тока $I$, соответствующий начальной фазе разряда. Рост $U$ и спад $I$ в интервале времени между $t_{U}$ и $t_{b d}$ соответствует отражению волны напряжения на изначально разомкнутом конце кабельной линии. Видно, что в интервале от 2 до $15 \mathrm{~ns}$ после пробоя ток $I$ линейно нарастает от 0.2 до $1 \mathrm{kA}$.

На рис. $2, a$ и $b$ представлены интерферограммы разряда, соответствующие обозначенным цифрами 1 и 2 временны́ отрезкам на осциллограммах, показанных на рис. 1.

Видно, что аналогично $[2,3]$ разряд развивается в микроканальной форме. В данном случае микроструктура разряда регистрируется на интерферограммах в виде модуляции интенсивности интерференционных полос. Заметный сдвиг полос в центральной области разрядного промежутка регистрируется после $6 \mathrm{~ns}$ (рис. 2). Максимального значения $2 \cdot 10^{19} \mathrm{~cm}^{-3}$ концентрация в этой области достигает при временах 20-30 ns. Диаметр канала разряда составляет примерно $0.3 \mathrm{~mm}$, количество микроканалов - около $N=100$. Оценка концентрации электронов в микроканале, радиус которого $r_{0}=5 \mu \mathrm{m}$, на 2 ns дает величину $n_{e} \approx 10^{17} \mathrm{~cm}^{-3}$. Измеренные значения радиусов микроканалов $r$ к моменту $15 \mathrm{~ns}$ достигают величины $r_{\max }=10 \mu \mathrm{m}$. К $30 \mathrm{~ns}$ канал разряда приобретает довольно четкую границу, после чего происходит его интенсивное радиальное расширение [3].

При проведении расчетов полагаем, что $r$ и $n_{e}$ во временно́м интервале от 2 до $15 \mathrm{~ns}$ растут линейно соответственно от $r_{0}$ до $r_{\max }$ и от $10^{17}$ до $1 \cdot 10^{19} \mathrm{~cm}^{-3}$.

Согласно [3], температура тяжелых частиц полагается высокой, что дает возможность рассматривать диссоциированный газ, компонентами которого являются атомарный азот и кислород (с долей $\alpha=0.2$ ). Введем эффективный коэффициент и потенциал ионизации атомарного воздуха как $k_{i o n}=\alpha k_{i o n}^{\mathrm{O}}+(1-\alpha) k_{i o n}^{\mathrm{N}}$ и $I_{i o n}=\alpha I_{i o n}^{\mathrm{O}}+(1-\alpha) I_{i o n}^{\mathrm{N}}$ соответственно. Здесь $k_{i o n}^{\mathrm{O}}$, $k_{i o n}^{\mathrm{N}}$ - скорость ионизации атомарного кислорода и азота, $I_{i o n}^{\mathrm{O}}, I_{i o n}^{\mathrm{N}}-$ потенциал ионизации атомарного кислорода и азота (в $\mathrm{eV})$.

Запишем уравнение для температуры электронов с учетом квазинейтральности

$$
\begin{aligned}
& \frac{3}{2} \frac{d T_{e}}{d t}=w E-\sum_{i} \frac{2 m_{e}}{M_{i}} k_{\text {elastic }}^{i} n^{i}\left(\frac{3}{2} T_{e}-\frac{3}{2} T_{i}\right) \\
& -\sum_{i} k_{e l}^{i} n^{i} I_{e l}^{i}-k_{\text {ion }} n\left(I_{\text {ion }}+3 T_{e}\right) \\
& -\sum_{i} k_{\text {ion_ex }}^{i} n_{\text {ex }}^{i} I_{\text {ion }}^{i}+k_{3} n_{e}^{2}\left(I_{\text {ion }}+3 T_{e}\right)-T_{e}^{2} \frac{d k_{3}}{d T_{e}} n_{e}^{2},
\end{aligned}
$$

где $t-$ время, $T_{e}, T_{i}-$ температуры электронов и $i$-й тяжелой частицы (в $\mathrm{eV})$; суммирование проводится по всем компонентам тяжелых частиц; $w=\frac{j}{e n_{e}}=\frac{I}{\pi r^{2} N e n_{e}}-$ дрейфовая скорость электрона; $E=\frac{j}{\sigma\left(T_{e}\right)}-$ напряженность поля; $m_{e}-$ масса электрона; $n-$ концентрация нейтральных частиц; $M_{i}, n^{i}-$ масса и концентрация $i$-й тяжелой частицы; $n_{e x}^{i}-$ концентрация частиц с возбужденным $i$-м электронным уровнем; $I_{e l}^{i}, I_{i o n}^{i}-$ энергия возбуждения и ионизации $i$-го электронного уровня (в $\mathrm{eV}), k_{\text {elastic }}^{i}-$ константа упругих потерь при столкновении электрона с соответствующей $i$-й тяжелой частицей (с ионами рассчитывалась в кулоновском приближении, для нейтральных частиц бралась как в [3]); $k_{i o n}, k_{e l}^{i}, k_{\text {ion ex }}^{i}-$ константы прямой ионизации, возбуждения и ионизации $i$-го электронного уровня (брались как в работе [3] и ссылках к ней $) ; k_{3}=\frac{10^{-27}}{\left.T_{e}[\mathrm{eV}]\right]^{4.5}} \mathrm{~cm}^{6} / \mathrm{s}-$ константа трехчастичной рекомбинации.

Электропроводность рассчитывалась по формуле типа Фроста [4]:

$$
\begin{aligned}
\sigma & =\frac{4}{3 \sqrt{\pi}} \frac{n_{e} e^{2}}{m_{e}\left(k_{\mathrm{B}} T_{e}\right)^{5 / 2}} \int_{0}^{\infty} \frac{\varepsilon^{3 / 2}}{v_{e a}+v_{e i} \gamma^{-1}} \\
& \times \exp \left(-\frac{\varepsilon}{k_{\mathrm{B}} T_{e}}\right) d \varepsilon\left[\Omega^{-1} \cdot \mathrm{m}^{-1}\right],
\end{aligned}
$$

где $e, m_{e}-$ заряд и масса электрона, $T_{e}-$ температура электронов (в $\mathrm{K}), k_{\mathrm{B}}-$ постоянная Больцмана, $v_{e a}$, 
$a$
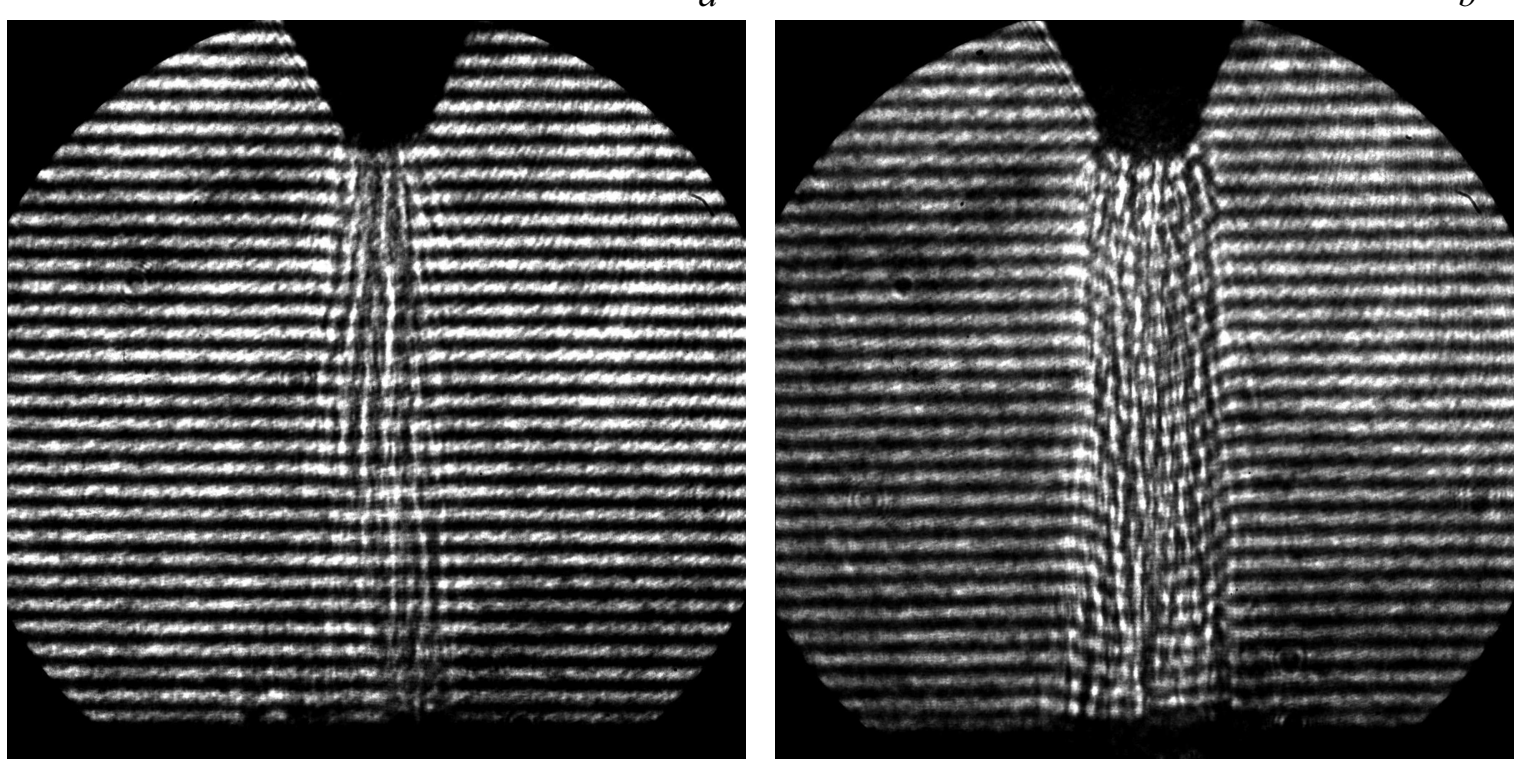

Рис. 2. Интерферограммы разряда в различные моменты времени относительно пробоя: $a-6 \mathrm{~ns}, b-17 \mathrm{~ns}$. Электрод-острие находится сверху.

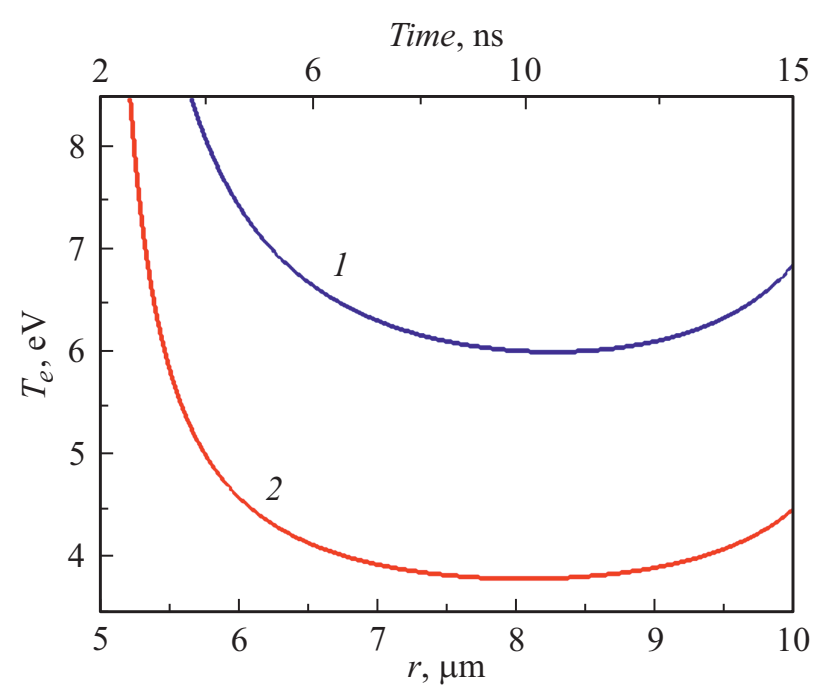

Рис. 3. Температура электронов $T_{e}$ в зависимости от радиуса микроканала $r .1$ - учет только прямой ионизации, 2 прямая и мгновенная ступенчатая ионизация.

$v_{e i}$ - частота столкновения электрона с атомами и ионами, $\gamma=0.582-$ коэффициент Спитцера для однократно ионизированного газа, $\varepsilon-$ энергия.

При этом в (1) полагаем, что расширение микроканала происходит за счет радиального движения первоначального газа (заключенного в микроканале в момент времени $2 \mathrm{~ns}$ ) без вовлечения в проводящую область новых порций окружающего воздуха.

Рассмотрим два предельных случая. В первом пренебрежем возбуждением электронных уровней и ступенчатой ионизацией, что дает оценку сверху для $T_{e}$. Для второго случая считаем, что, как только атом перешел в возбужденное состояние, он мгновенно ионизируется, соответственно получим нижний передел $T_{e}$.

На рис. 3 приведены решения уравнения (1) для двух предельных случаев $T_{e}$. Верхняя шкала соответствует времени после пробоя, в течение которого происходит расширение микроканала и нарастание тока. В качестве начальных значений выбирались два: $T_{e}^{0}=1$ и $10 \mathrm{eV}$. При этом, как показали расчеты, уже через время $\sim 0.1 \mathrm{~ns}$ решение не зависит от $T_{e}^{0}$.

Таким образом, на основании проведенных расчетов можно полагать, что в микроканале средняя температура электронов находится в интервале $T_{e}=4-8 \mathrm{eV}$. Полученные результаты согласуются с экспериментальными данными по измерению $T_{e}$ в искровом разряде в азоте атмосферного давления в миллиметровом промежутке при временах 10-20 ns после приложенного напряжения, где были получены значения $T_{e}=5-6 \mathrm{eV}$ [5]. Плотность тока есть $j=\frac{I}{\pi r^{2} N}=10^{6}-10^{7} \mathrm{~A} / \mathrm{cm}^{2}$, проводимость составляет $\sigma \sim 10 \Omega^{-1} \cdot \mathrm{cm}^{-1}$, а напряженность электрического поля находится на уровне $E \sim 300 \mathrm{kV} / \mathrm{cm}$. Следует отметить, что в рассматриваемых условиях вычисленная температура электронов в микроканалах относительно высокая. Полученные значения температуры, например, близки или превосходят температуру в филаментах (микроразрядах) барьерного разряда в воздухе при аналогичных значениях $E / p$, однако степень ионизации в барьерном разряде гораздо меньше [6].

Полученные экспериментальные данные и расчеты, выполненные на их основе, свидетельствуют о том, что реализация микроструктурированного разряда обусловливает в его начальной фазе (в течение $15 \mathrm{~ns}$ после пробоя) относительно высокие значения средней темпера- 
туры электронов (до $8 \mathrm{eV}$ ) в совокупности с достаточно большой степенью ионизации (до полной однократной). Представленные данные могут быть полезны как для понимания физики процессов в начальной фазе разрядов в плотных газах, так и для практических приложений ввиду сильной зависимости от температуры и степени ионизации кинетики плазмохимических реакций.

\section{Финансирование работы}

Работа выполнена при финансовой поддержке Российского фонда фундаментальных исследований (грант № 20-08-01069a).

\section{Конфликт интересов}

Авторы заявляют, что у них нет конфликта интересов.

\section{Список литературы}

[1] Д.В. Белоплотов, В.Ф. Тарасенко, Д.А. Сорокин, М.И. Ломаев, Письма в ЖЭТФ, 106 (10), 627 (2017).

[2] E.V. Parkevich, M.A. Medvedev, A.I. Khirianova, G.V. Ivanenkov, A.S. Selyukov, A.V. Agafonov, K.V. Shpakov, A.V. Oginov, Plasma Sources Sci. Technol., 12 (28), 125007 (2019).

[3] K.I. Almazova, A.N. Belonogov, V.V. Borovkov, Z.R. Khalikova, G.B. Ragimkhanov, D.V. Tereshonok, A.A. Trenkin, Plasma Sources Sci. Technol., in press (2020). https://doi.org/10.1088/1361-6595/aba8cc

[4] И.Т. Якубов, УФН, 163 (5), 35 (1993).

[5] H. Albrecht, W.H. Bloss, W. Herden, R. Maly, B. Saggau, E. Wagner, SAE, paper 770853 (1977).

[6] В.Г. Самойлович, В.И. Гибалов, К.В. Козлов, Физическая химия барьерного разряда (Изд-во МГУ, М., 1989). 